\title{
Secreted Protein Acidic and Rich in Cysteine in Ocular Tissue
}

\author{
Kurt Scavelli, Ayan Chatterjee, and Douglas J. Rhee
}

\begin{abstract}
Secreted protein acidic and rich in cysteine (SPARC), also known as osteonectin or BM-40, is the prototypical matricellular protein. Matricellular proteins are nonstructural secreted proteins that provide an integration between cells and their surrounding extracellular matrix (ECM). Regulation of the ECM is important in maintaining the physiologic function of tissues. Elevated levels of SPARC have been identified in a variety of diseases involving pathologic tissue remodeling, such as hepatic fibrosis, systemic sclerosis, and certain carcinomas. Within the eye, SPARC has been identified in the trabecular meshwork, lens, and retina. Studies have begun to show the role of SPARC in these tissues and its possible role, specifically in primary open-angle glaucoma, cataracts, and proliferative vitreoretinopathy. SPARC may, therefore, be a therapeutic target in the treatment of certain ocular diseases. Further investigation into the mechanism of action of SPARC will be necessary in the development of SPARC-targeted therapy.
\end{abstract}

\section{Introduction}

acreted PRotein acidic and rich in cysteine (SPARC), $\checkmark$ also known as osteonectin or BM-40, is a calciumbinding matricellular glycoprotein. The matricellular proteins are nonstructural secreted glycoproteins that facilitate cellular control over their surrounding extracellular matrix (ECM). ${ }^{1}$ The matricellular family includes SPARC, myocilin, connective tissue growth factor, thrombospondin-1 and thrombospondin-2 (TSP-1 and -2), tenascin-C and tenascin$\mathrm{X}, \mathrm{SC} 1 /$ hevin, and osteopontin. ${ }^{2-5}$ In general, matricellular proteins provide an integration between cells and the ECM by providing an interaction between structural proteins and signaling molecules, such as cytokines and matrix proteases. ${ }^{6}$

SPARC is the prototypical matricellular protein. It was first identified in the bone in 1981 where it was initially referred to as osteonectin. ${ }^{7}$ SPARC is a highly conserved protein across multiple species and shares greater than $70 \%$ amino acid sequence homology across mammalians, amphibians, and avians, indicating its biologic importance. ${ }^{8}$ It is highly expressed in most embryonic tissues, however, in adult tissue, SPARC has a narrower distribution, ${ }^{9,10}$ but is highly expressed in tissues undergoing extensive remodeling, such as the bone and gut. However, any tissue with the need to remodel ECM, for example, during wound repair, can increase SPARC expression. ${ }^{9-11}$ It has multiple functions in vitro, such as ECM modification, disruption of cell adhesion, cell cycle inhibition, cell differentiation, morphogenesis, and migration. ${ }^{12-19}$

A particularly important function of SPARC is its role in ECM turnover. SPARC is highly expressed in tissue undergoing a high amount of tissue remodeling. Within these tissues, SPARC can be found in abundance within connective tissue and basement membranes. ${ }^{20,21}$ Elevated SPARC expression has been described in a variety of diseases involving pathologic tissue remodeling: cholangitis, hepatic fibrosis, renal interstitial fibrosis, systemic sclerosis, osteoporosis, and certain carcinomas. ${ }^{22-28}$ Conversely, SPARC suppression decreases the expression of multiple collagens and the tissue inhibitor of metalloproteinase (TIMP)-3. ${ }^{29,30}$ SPARC has been implicated in enhancing fibronectin-mediated ECM assembly through an interaction with the integrin-linked kinase (ILK). ${ }^{31,32}$ Mice with single-gene deletions of Sparc had accelerated dermal wound closure, decreased levels of dermal collagen, and decreased bone mineral density. ${ }^{33-35}$

SPARC has an important relationship with transforming growth factor-beta (TGF- $\beta$ ), which is known to be involved in ECM deposition. ${ }^{36,37}$ In human fibroblasts, TGF- $\beta$ has been shown to increase the SPARC mRNA through a posttranscriptional mechanism. ${ }^{38}$ SPARC has also been shown to be influential in modulating the expression of TGF$\beta 1 .^{39,40}$ SPARC-null cells have decreased TGF- $\beta 1$ mRNA, and the addition of exogenous SPARC results in return to the normal mRNA level of wild-type cells. ${ }^{41,42}$ Elucidating

Department of Ophthalmology and Visual Sciences, University Hospitals Eye Institute, Case Western Reserve University School of Medicine, Cleveland, Ohio. 
the regulatory interactions between TGF- $\beta 1$ and SPARC may lead to insights into the disease entity of pseudoexfoliation glaucoma, in which TGF- $\beta 1$ is significantly increased. There is particular interest in the association of SPARC with TGF- $\beta 2$, given its known significance in primary open-angle glaucoma (POAG). ${ }^{43,44}$ TGF- $\beta 2$ is known to upregulate SPARC expression, and SPARC may represent a key node in TGF- $\beta 2$-mediated ocular hypertension. ${ }^{39}$

Across all species, SPARC is encoded by a single gene. ${ }^{45,46}$ Following translation, SPARC is a $32 \mathrm{kDa}$ protein. It is post-translationally glycosylated, and the glycosylation structure varies depending on the tissue in which it is expressed. ${ }^{45}$ Following glycosylation, SPARC has a molecular weight of $\sim 43 \mathrm{kDa} .{ }^{47}$ The protein comprises 3 distinct domains that provide the properties of SPARC (Fig. 1). Domain I functions to bind calcium ions and hydroxyapatite, making it an important domain for SPARC's role in the mineralization of bone. ${ }^{48,49}$ Domain II is referred to as the follistatin-like (FS) domain. It is within the FS domain that SPARC receives its glycosylation, specifically at Asn99. A wide variety of glycosylation patterns have been demonstrated in SPARC expressed in different tissue types. ${ }^{50}$ The composition of SPARC's glycosylation is important for the functionality of the protein within a particular tissue. ${ }^{51}$ In particular, the binding affinity of SPARC for collagen I can vary as much as a $100 \times$ depending on the structural variability of SPARC's glycosylation. ${ }^{50}$ Domain III is referred to as the extracellular calcium-binding (EC) domain. The EC domain contains a pair of EF-hands that have highaffinity calcium-binding sites. Another important feature of the EC domain is a binding epitope that has affinity for collagen types I, IV, and V. ${ }^{52}$ Interestingly, SPARC exhibits a 7- to 20-fold increase in binding affinity for collagens I, $\mathrm{IV}$, and $\mathrm{V}$, following cleavage by matrix metalloproteinases (MMPs). ${ }^{53}$ It is believed that cleavage of SPARC by MMPs explains the 15-fold greater binding affinity of tumor tissuederived murine SPARC for collagen IV, since murine SPARC is endogenously cleaved at a site 1 residue away from the cleavage site of MMPs. ${ }^{21}$ Enzymatic cleavage may, therefore, be another mechanism of post-transcriptional modification of SPARC.

The binding of calcium ions to SPARC is vital to the structure and function of the protein. Calcium binding to SPARC's EC domain results in an increase in the $\alpha$-helicity of the protein and this conformational change increases SPARC's affinity for collagen. ${ }^{48,54,55}$ The concentration of calcium in the environment is, therefore, very important in regulating SPARC's activity. One study showed that SPARC can act as a matrix scavenger chaperone in fibroblasts by binding collagen in the high-calcium environment of the ECM and releasing collagen in the low-calcium environment of the cytosol. ${ }^{56}$ It has been demonstrated that SPARC has general chaperone-like molecular activity by its ability to prevent the thermal aggregation of alcohol dehydrogenase in vitro. ${ }^{57}$ Given SPARC's regulation of ECM deposition and turnover in the context of its known binding to ECM proteins, such as collagens, SPARC may play an important role as a chaperone for ECM proteins.

The adult eye expresses high levels of SPARC compared to the developing eye. ${ }^{58}$ SPARC has been found in the cornea, ciliary epithelium, retinal pigment epithelium, aqueous and vitreous humor, trabecular meshwork (TM), and lens epithelial cells (LECs). Our laboratory has focused on SPARC's involvement in modulating outflow facility through the TM, but there is growing evidence for SPARC's importance in the lens and retina of the eye as well. This article represents a comprehensive review on the progress made in understanding the role of the matricellular protein SPARC in the TM, lens, and retina of the eye.

\section{Trabecular Meshwork}

POAG is one of the leading causes of irreversible blindness in the world, affecting more than 70 million people. ${ }^{59-61}$ Elevated intraocular pressure (IOP) is the greatest risk factor contributing to POAG. ${ }^{62}$ The elevated IOP develops as a result of increased resistance to aqueous humor outflow through the trabecular outflow pathway. ${ }^{63-67}$ The trabecular outflow pathway is the primary conduit for drainage of
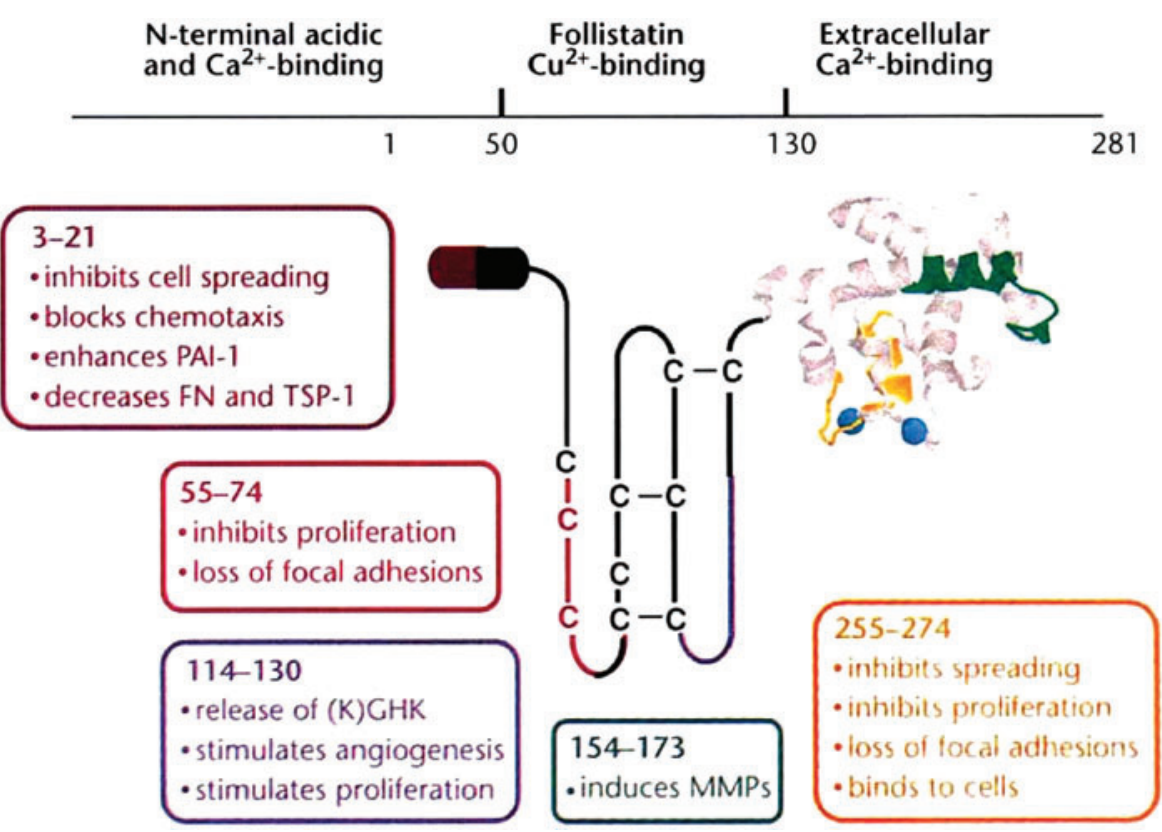

FIG. 1. SPARC comprises 3 distinct structural domains. Domain I (N-terminal acidic and calciumbinding) functions to bind calcium and hydroxyapatite (adapted, with permission, from Sage $1997 ;^{68}$ Copyright ( 1997 Nature Publishing Group). Domain II (follistatin-like domain) is where SPARC undergoes glycosylation and binds collagens. Domain III (extracellular calciumbinding domain) is the main calcium-binding domain for SPARC. PAI, plasminogen activator inhibitor; FN, fibronectin; TSP, thrombospondin; C, cysteine; MMPs, matrix metalloproteinases; SPARC, secreted protein acidic and rich in cysteine. Color images available online at www.liebertpub.com/jop 
aqueous humor $(60 \%-95 \%)$ in the eye (Fig. 2). Within the trabecular outflow pathway, the site of highest outflow resistance is the TM, specifically the juxtacanalicular tissue (JCT) ${ }^{69-73}$ The TM is one of the anatomically important locations in the pathogenesis of POAG. The characteristic pathological finding within the TM of untreated diseased eyes is an increase in sheath-derived plaques. ${ }^{74}$ Sheath-derived plaques comprise many ECM proteins, such as fibronectin, laminin, collagen I, III, IV, V, and VI. ${ }^{75}$ Along with the increase in sheath-derived plaques within the TM, there is an increase in the intrinsic stiffness of the tissue. ${ }^{76}$ These findings strongly suggest that POAG is a result of aberrant regulation of the ECM within the TM. We believe that matricellular proteins, which are involved in the regulation of ECM equilibrium, are influential in the altered ECM observed in POAG.

SPARC is one of the most highly expressed genes in primary cultures of human TM cells and in TM cells that undergo physiological stress. ${ }^{77-79} \mathrm{TM}$ cells cultured on

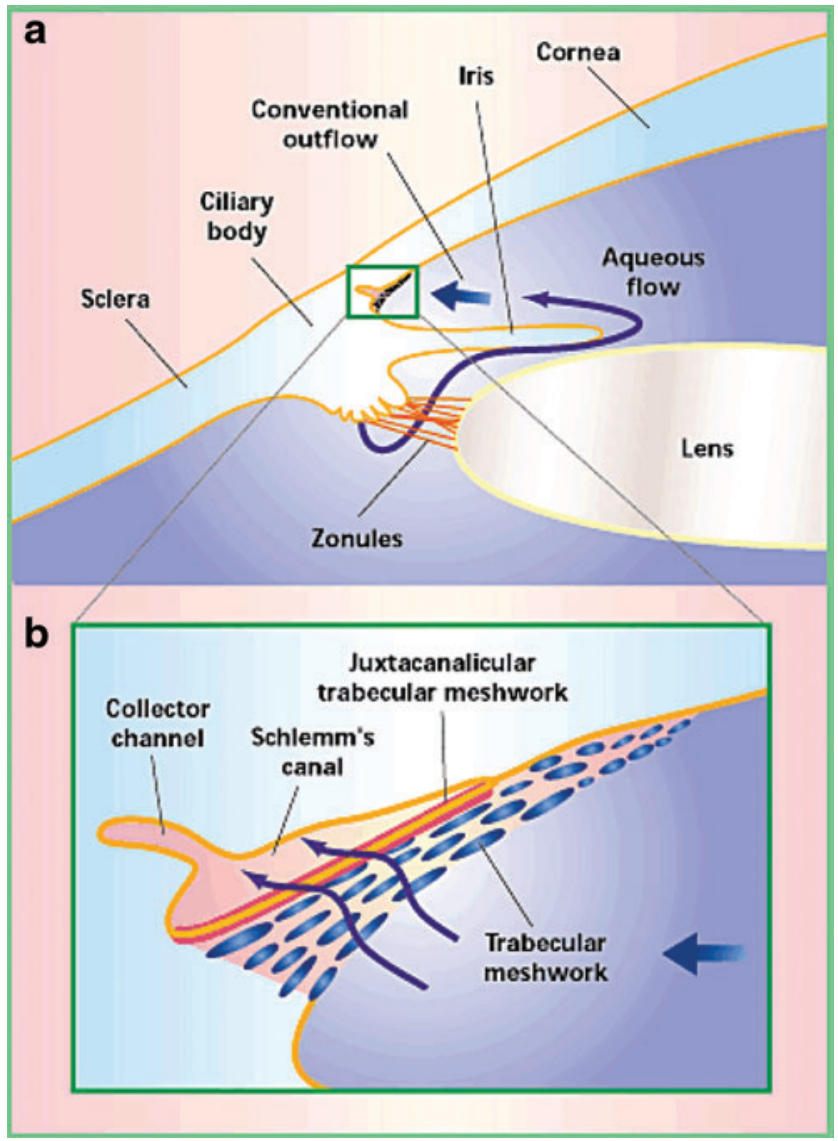

FIG. 2. (a) $\mathrm{AH}$ is produced from the ciliary body in the posterior chamber and passes into the anterior chamber through the pupil (adapted, with permission, from Tomarev 2001;80 Copyright (C) 2001 Nature Publishing Group). The majority (60\%-95\%) of the AH leaves the eye through the TM located at the iridocorneal angle in the anterior chamber. (b) An enlargement from (a) that shows the flow of AH through the trabecular outflow pathway. The juxtacanalicular TM is the region of highest resistance in the TM. After traversing the TM, AH drains into Schlemm's canal and subsequently collector channels. AH, aqueous humor; TM, trabecular meshwork. Color images available online at www.liebertpub .com/jop synthetic hydrogels stimulating the stiffness found in glaucomatous TM exhibited significantly increased SPARC mRNA expression compared to those cultured on hydrogels that exhibit the lower stiffness representative of normal TM. ${ }^{81}$ We have demonstrated that SPARC-null mice exhibit 15\%-20\% lower IOP with greater amounts of TM utilized for aqueous humor outflow than their wild-type counterparts. ${ }^{82,83}$ SPARC-null mice have a decrease in the collagen fibril diameter within the JCT of the TM, consistent with that observed in the dermis of SPARC-null mice. ${ }^{33,83}$ These data suggest a mechanism by which IOP is reduced in SPARC-null mice. Given SPARC's effect on collagen fibril diameter, variation in the spatial expression of SPARC throughout the TM may be a mechanism for the generation of segmental flow that occurs in the human and murine eye. ${ }^{84}$ SPARC overexpression in human TM cells, conversely, resulted in elevated IOP in an ex vivo anterior chamber perfusion chamber model. ${ }^{85}$ Overexpression of SPARC within TM cell cultures and within the JCT of perfused anterior segments resulted in elevated protein levels of fibronectin and collagens I and IV. Interestingly, quantitative PCR did not detect a significant elevation of mRNA for any of these proteins. ${ }^{85}$ This finding indicates that the mechanism for SPARC's influence on ECM proteins is not a simple increase in production. SPARC may act as a protective chaperone to stabilize ECM proteins from native enzymatic degradation.

SPARC has previously been shown to have chaperone-like activity. ${ }^{57}$ It is a stress-induced protein and its secondary structure is stable up to $50^{\circ} \mathrm{C} .{ }^{86,87} \mathrm{ECM}$ turnover is a dynamic process that involves a balance between the deposition of collagens and degradation by various MMPs. Given its $\mathrm{Ca}^{2+}$ dependent binding to collagens I and IV, SPARC may play a role in preventing MMP-mediated degradation of collagens I and IV. ${ }^{52,53}$ Preliminary in vitro data from our laboratory support this hypothesis. ${ }^{88}$ There is also evidence that SPARC may regulate ECM turnover by decreasing the degradative process (ie, MMPs). Outflow facility studies have demonstrated the importance of MMPs in modulating the balance between ECM degradation and synthesis in regulating IOP. ${ }^{64}$ Overexpression of SPARC in human TM cells results in a decrease in MMP-9 protein levels and an increase in TIMP- $1 .{ }^{85}$ A similar finding was observed in medulloblastoma tumor cells overexpressing SPARC, which demonstrated a decrease in pro-MMP-9 expression. ${ }^{89}$ Taken together, the evidence supports that SPARC regulates increased ECM deposition by acting as a chaperone to prevent the degradation of ECM proteins and also by decreasing the expression and activity of MMPs.

An important and well-established finding in eyes with POAG is an elevation of TGF- $\beta 2$ in the aqueous humor compared to age-matched individuals without glaucoma. ${ }^{43,44,90}$ TGF- $\beta 2$ mediates fibrosis and ECM deposition in many disorders. ${ }^{91-93}$ In vitro, TGF- $\beta 2$ stimulates TM cells to synthesize and secrete certain structural and regulatory ECM proteins. ${ }^{94-97}$ In perfused human anterior chambers, administration of exogenous TGF- $\beta 2$ resulted in an increase in IOP ${ }^{98,99}$ Furthermore, in response to TGF- $\beta 2$ stimulation, SPARC is the most highly expressed protein by TM cells. ${ }^{106}$ When SPARC is administered exogenously to TM cells, there is no effect on TGF- $\beta 2$ in cultured TM cells. SPARC, therefore, appears to be a downstream mediator of TGF- $\beta 2 .{ }^{39}$ In TM cells, TGF- $\beta 2$ upregulates SPARC through Smad3, 
JNK, and p38 pathways (Fig. 3). SPARC-null mice display significantly attenuated TGF- $\beta 2$-mediated ocular hypertension, ${ }^{40}$ suggesting that SPARC is essential to the regulation of TGF- 32 -mediated ocular hypertension.

\section{Lens}

Over the past 2 decades, there has been emerging evidence that SPARC plays a pivotal role in maintaining the structural integrity of the lens capsule. Evidence for this first came to light with the observation that SPARC-null mice demonstrated $100 \%$ penetrance for the development of cataracts, but otherwise had normal growth, fertility, and viability. ${ }^{101}$ SPARC-null mice start to develop posterior cortical opacities, evident by a slit lamp, beginning as early as 6 weeks after birth. ${ }^{12,101}$ On histopathology, there is evidence of impaired fiber cell differentiation, vacuolization in the equatorial region, and posterior displacement of the lens nucleus. By 3.5 months, $46 \%$ of SPARC-null mice exhibit mature cataracts. With further progression of the cataract around 5-8 months, severe complications develop, such as rupture and displacement of the lens into the anterior chamber. Although the earliest signs of cataract formation cannot be seen on a slit lamp till around 6 weeks, electron microscopy of the SPARC-null lens at 1 week shows the presence of sparse membranous projections from the basal surface of the LECs into the capsule. ${ }^{102}$ By the time there is the development of a posterior cortical opacity, large and abundant projections can be seen throughout the capsule (Fig. 4). Further immunohistologic analysis of these projections demonstrates the presence of actin. Given the importance of actin filaments in shaping the posterior surface of the lens and their interaction with integrins of the basement membrane of the lens fiber cells, integrins may be a mediator in SPARC's maintenance of lens capsule integrity. ${ }^{102}$

SPARC is known to interact with integrin $\beta 1$ to activate ILK to mediate cell survival in LECs. ${ }^{103}$ ILK activation by SPARC may also be important in maintaining the LEC and basement membrane interface. Similar membranous projections have been documented in the lens capsule of aging mice and humans; however, their localization was only in the equatorial region of the lens, whereas SPARC-null mice have projections throughout the entirety of the capsule. ${ }^{104,105}$ Other histological findings of SPARC-null lenses are incomplete fiber cell elongation, retention of nuclear debris within fiber cells, swollen anterior cortical fibers, and thickening of the entire capsule. ${ }^{12}$ Overall, these findings demonstrate that SPARC is integral in maintaining the transparency of the lens, specifically through aiding in fiber cell differentiation and maintenance of the structural integrity of the LEC and capsule interface. SPARC's maintenance of the LEC and capsule interface provides more evidence of its role in regulating cell-matrix interactions that have already been demonstrated in other tissues.

Within the lens, SPARC expression has been localized to LECs with expression ending at the equatorial bow where differentiation into fiber cells occurs. ${ }^{12,106} \mathrm{~A}$ key feature of the transparency of the mammalian lens is the presence of transparent fiber cells that are devoid of organelles and nuclei. As LECs age, they enter into a state of quiescence and migrate toward the equatorial region of the lens. At the equatorial region, the morphological differentiation of LECs into fiber cells occurs. ${ }^{107}$ Each step in the process of fiber cell formation within the lens is regulated by the interaction of ECM proteins in the capsule with LECs. ${ }^{108,109}$ Outside of the LECs, SPARC is present in the aqueous and vitreous humor and could be another source of SPARC for the avascular lens. ${ }^{110}$ There is evidence that components of the

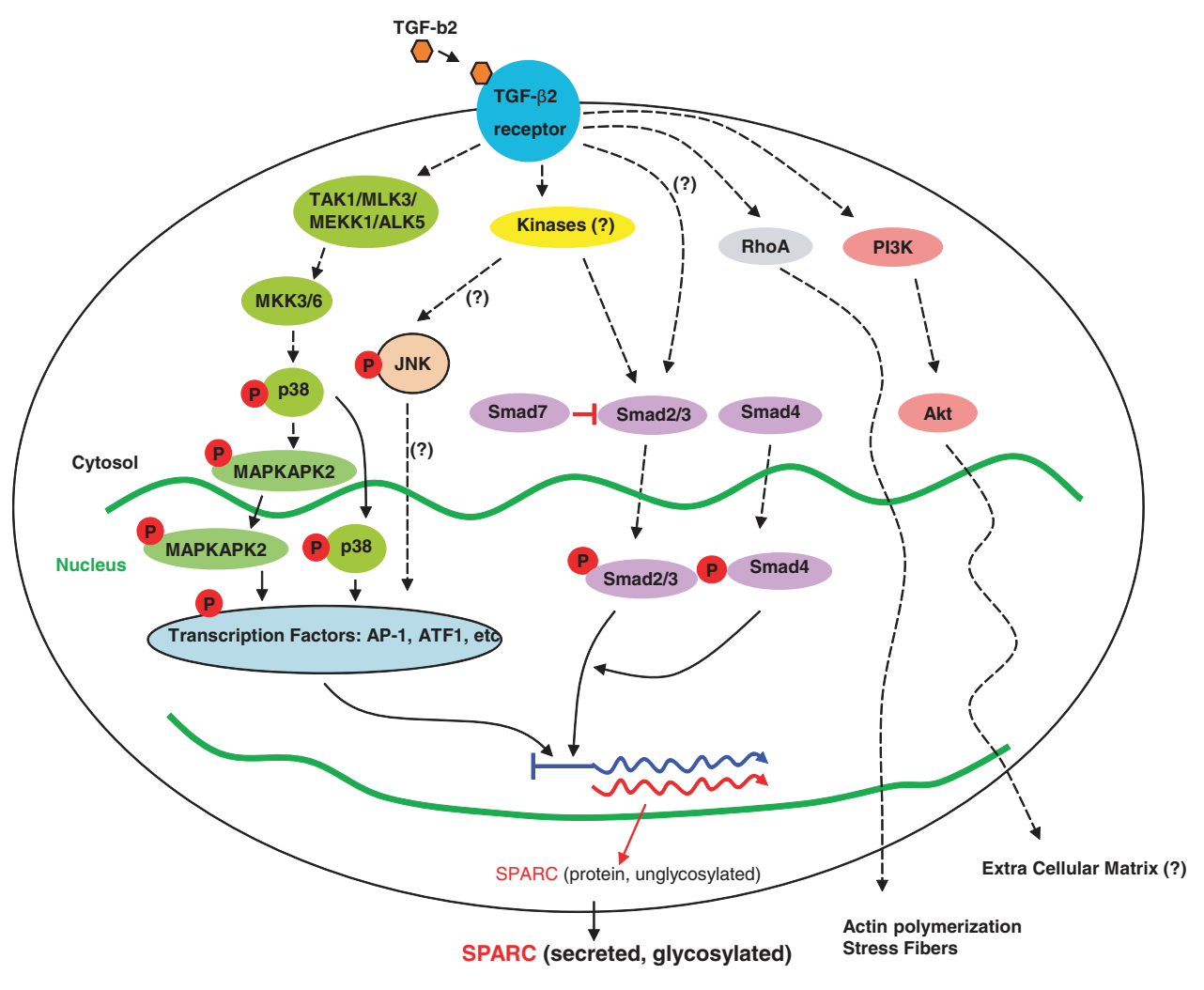

FIG. 3. A summary of TGF$\beta 2$ signaling pathways involved in the regulation of SPARC in TM cells (adapted, with permission, from Kang et al. 2013 39; Copyright (C) 2013 Association for Research in Vision and Ophthalmology). TGF- $\beta 2$, transforming growth factor-beta 2. Color images available online at www.liebertpub.com/jop 
FIG. 4. Transmission EM of LECs and lens capsule interface. All images are of the same region of the lens (adapted, with permission, from Yan et al. 2002; Copyright (C) 2002 by The Company of Biologists Ltd.). (A) One-month-old SPARC ${ }^{+/+}$ lens capsule displaying a normal LEC and capsule interface. (B) One-month-old SPARC $^{-/-}$lens capsule. Protrusions of lens fiber cells are beginning to invade into the lens capsule (Arrows show the protrusions). (C) Three-month-old SPARC $^{+/+}$lens. (D) Three-monthold SPARC ${ }^{-l-}$ lens. There is extensive infiltration of lens fibers into the lens capsule. Scale bar, $5 \mu \mathrm{m}$. LEC, lens epithelial cell.
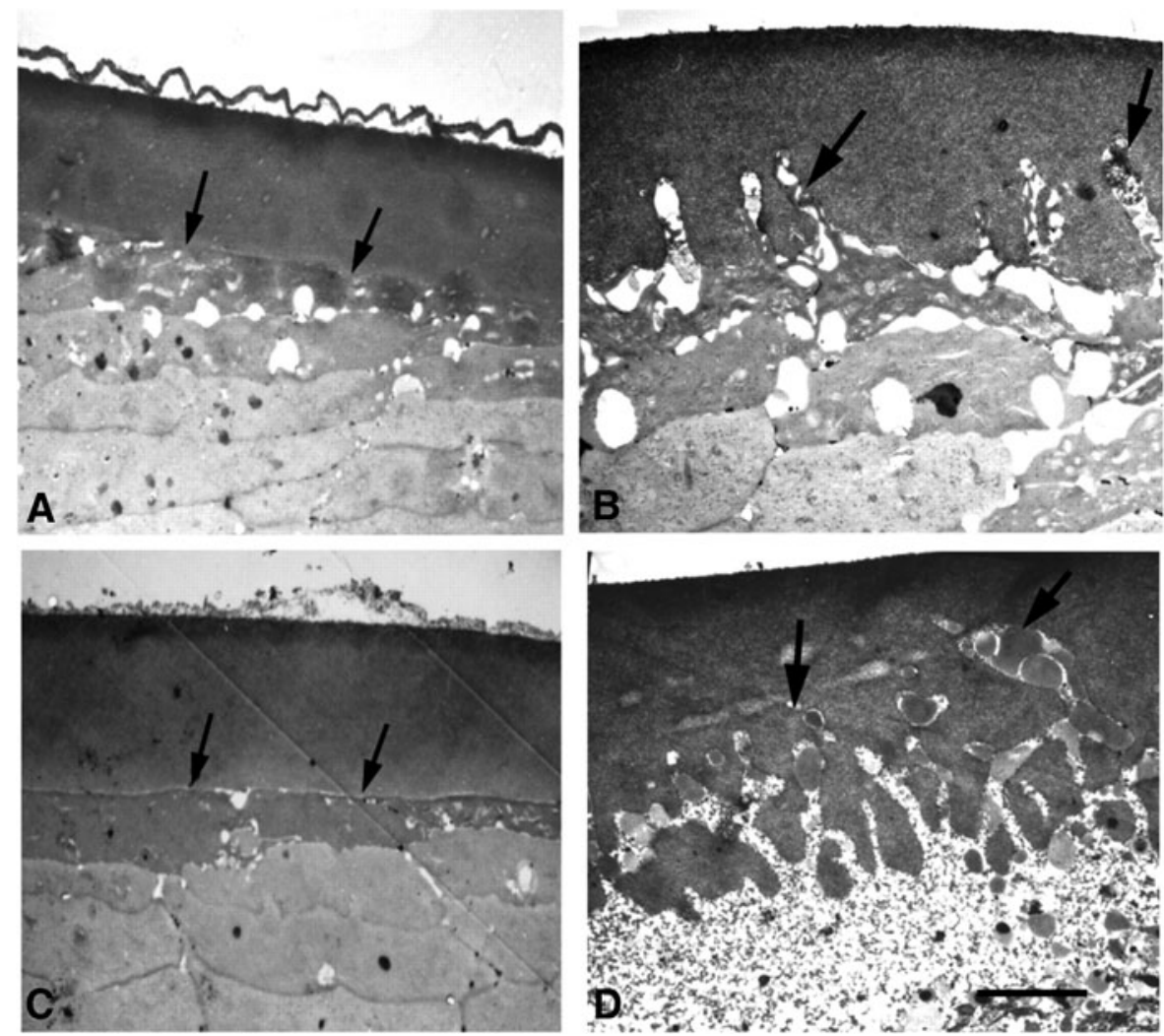

vitreous humor can affect LEC development and lens fiber differentiation. ${ }^{112,113}$ The vitreous humor may, therefore, be important in maintaining lens transparency, and vitreous SPARC could be one of the key mediators. Given SPARC's role in cell-matrix interactions and the decrease in fiber cell differentiation demonstrated in SPARC-null lenses, SPARC presents as a possible intermediary for the influence of ECM on fiber cell development.

The lens capsule is a thick basement membrane and it is made up of $90 \%$ collagen IV along with laminin, fibronectin, and other ECM components. ${ }^{102}$ Its structural morphology is important in maintaining the refractive nature of the lens. Since the lens is an avascular structure, water and ions must diffuse from the aqueous humor through the capsule to nourish the LECs. Regulation of the organization of the capsule is important in maintaining adequate and appropriate filtration and permeability. In SPARC-null lenses, there is alteration of the organization of collagen IV within the capsule resulting in disruption of the normal physiologic barrier. As a result, the lenses have increased water and ion influx causing osmotic swelling of the fiber cells. ${ }^{114}$ SPARC, thus, is important in the organization of ECM proteins within the lens capsule.

In the human lens, SPARC mRNA expression is localized to the LECs, with higher levels of protein detected in the peripheral LEC compared to the central LECs. In a comparison between age-related cataractous lenses and normal lenses, increased expression and protein levels of SPARC were found in age-related cataractous lenses. ${ }^{115,116}$ The increased levels of SPARC in cataractous lenses may be a stress response to the cataract in the lens. Elevated SPARC levels may be required to maintain the structural integrity of the lens, although the exact mechanism remains unclear.

\section{Retina}

SPARC was first identified in the adult central nervous system (CNS) in 1994 when Mendis et al. demonstrated that SPARC mRNA and protein were present in normal adult brain tissue. ${ }^{117,118}$ SPARC has been identified in the bovine, quail, chicken, and monkey retina. ${ }^{58,119-121}$ In the bovine retina, SPARC is localized to the soma and axon of ganglion cells, astrocytes, Muller glial cells, and endothelial cells of capillaries. ${ }^{58}$ Interestingly, the Muller and ganglion cells of the retina are known to express TGF- $\beta .^{122}$ Given SPARC's known involvement with TGF- $\beta$, this could be a pathway for SPARC's role in the retina. Another finding in the bovine retina was a higher expression of SPARC observed in the adult retinal tissue compared to the newborn retinal tissue, suggesting the protein's involvement in physiologic maintenance. ${ }^{58}$ Studies of SPARC in the monkey retina demonstrate that SPARC is synthesized primarily in the retinal pigment epithelial (RPE) and then transported to the outer plexiform layer. SPARC mRNA expression was found to be 10 -fold greater in the RPE of the macula compared to the peripheral retina. ${ }^{121}$ Its localization to an environment with a high concentration of synapses suggests that SPARC may play a role in synaptogenesis. In rat retinal ganglion cells (RGCs), astrocyte-secreted SPARC-like 1 (also known as hevin) was shown to induce synaptic formation in vitro. SPARC, alternatively, strongly inhibited hevin-induced synaptogenesis in this model. ${ }^{123}$ The interplay of these 2 matricellular proteins presents an interesting method of regulating synaptic formation and plasticity, as well as providing synaptic maintenance in the CNS.

In humans, SPARC is expressed and secreted by human retinal pigment epithelial (HRPE) cells. ${ }^{12,125}$ The cell 
density of HRPE cells influences the expression of SPARC, with a higher density of HRPE cells resulting in SPARC comprising a greater percentage of protein production. ${ }^{125}$ In HRPE cells, SPARC is primarily secreted in a basolateral orientation. Within the retina, the basal surface of HRPE cells is adherent to Bruch's membrane. Bruch's membrane comprises many ECM proteins such as proteoglycans (heparin and chondroitin sulfate), collagens (types I, III, IV, V, VI), laminin, and fibronectin. ${ }^{126}$ SPARC's localization to the basal surface of HRPE cells and known involvement in ECM regulation suggest that SPARC may regulate the adhesion of HRPE cells to the Bruch's membrane.

A disease model that has been used to investigate SPARC's role in regulating the interaction between HRPE cells and Bruch's membrane is proliferative vitreoretinopathy (PVR). PVR is characterized by the development of the epiretinal membrane (ERM) or subretinal membrane (SRM) that forms following surgical correction of a retinal detachment. In PVR, RPE cells detach from Bruch's membrane and migrate to PVR membranes where they dedifferentiate into a fibroblast-like phenotype. ${ }^{127}$ This dedifferentiation of RPE cells results in alteration of their surrounding ECM. ${ }^{127}$ SPARC has been detected in both the ERMs and SRMs of PVR. ${ }^{128}$ Other components of the matrix of PVR are collagens, elastic fibers, laminin, fibronectin, and other matricellular proteins (TSP-1 and tenascin).

When RPE cells first detach from Bruch's, they appear rounded and subsequently proliferate and migrate to PVR membranes. ${ }^{126}$ Matricellular proteins such as SPARC have wellestablished functions involving the regulation of adhesion, proliferation, and migration. ${ }^{1}$ Thus, SPARC may be a key initiator in the cascade of PVR. Migratory RPE cells of PVR membranes were identified by their detection with the monoclonal antibody RGE53; RGE53 detects cytokeratins 18 and 19, which are known markers for in vitro HRPE cell mobility. ${ }^{129}$ RGE53 ${ }^{+}$cells in PVR membranes were shown to colocalize with SPARC and TSP-1, but not with tenascin. ${ }^{126}$ This association suggests that SPARC and TSP-1 could be initiators of RPE migration in PVR. Follow-up studies looking at the effects of exogenous SPARC on proliferation and migration assays of HRPE cells demonstrated that SPARC has antiproliferative effects on HRPE cells in vitro. ${ }^{130}$ In this study, however, the exogenous SPARC was platelet derived and SPARC is known to have different glycosylation and function depending on what tissue it is expressed in. Given that only $10 \%$ of retinal detachments are complicated by PVR, susceptibility to development could be due to an individual's relative expression of matricellular proteins, specifically SPARC and TSP-1.

PVR membranes can develop in cases of diabetic retinopathy as well; however, they vary from PVR membranes developing subsequent to surgical correction, in that they are vascular and contain minimal RPE cells. ${ }^{126,131}$ A low concentration of RPE cells in PVR membranes of diabetic retinopathy makes it less likely that SPARC is influential in the development of the membranes. Further analysis, however, is needed to address this association.

SPARC has also been implicated in other retinal diseases, such as age-related macular degeneration (AMD). In HRPE cell cultures that were derived from donors with AMD and age-matched healthy donors, secreted proteome profiling demonstrated that there was a 2-fold decrease in SPARC in AMD RPE cells. ${ }^{132}$ The authors proposed that given SPARC's antiangiogenic effect, a decrease in the production may result in the increase in angiogenesis seen in AMD.
SPARC has also been identified as a Schwann cellsecreted factor that promotes RGC survival and outgrowth through Akt and Erk1/2 phosphorylation. ${ }^{133,134}$ In an optic nerve transection rat model, there was a 1.8-fold increase in Sparc mRNA expression of RGCs following transection. ${ }^{135}$ It is, therefore, possible that SPARC confers some regenerative benefit to RGCs.

\section{Conclusions}

Matricellular proteins have long been recognized as important regulators of cell-ECM interactions. More recently, their role in influencing cell phenotype and the pathogenesis of certain diseases has become evident. SPARC is the prototypical matricellular protein and has a relatively high level of expression in the adult eye. In the TM, SPARC regulates ECM homeostasis and aqueous outflow. SPARC's influence on TGF- $\beta 2$-mediated ocular hypertension provides a strong disease link to POAG pathogenesis. The $100 \%$ penetrance of cataracts in SPARC-null mice highlights the importance of the protein in sustaining lens transparency, most likely through maintenance of the LEC and capsule interface. In the retina, there is evidence that SPARC may function to maintain the RPE-Bruch's membrane interface. The large variety of roles that SPARC plays in this relatively small organ demonstrate the versatility of the protein. In general, SPARC's specific function appears to be different depending on the tissue in which it is expressed. The glycosylation pattern of SPARC is known to be tissue specific and is believed to influence the function of the protein. Further studies should attempt to characterize the glycosylation of SPARC from the TM, lens, and retina.

Overall, SPARC demonstrates important physiologic roles throughout the eye, and there is growing evidence to implicate SPARC in a number of ocular pathologies. SPARC may, therefore, be a therapeutic target in the treatment of ocular diseases, such as POAG. Inhibition of SPARC could present a method of decreasing TGF- $\beta 2$-mediated ocular hypertension believed to be integral in the pathogenesis of POAG. No small molecules, however, are currently known to bind to SPARC at the moment. Further investigation into the mechanism of action of SPARC will be necessary in the development of SPARC-targeted therapy.

\section{Acknowledgment}

Supported by: National Eye Institute EY 019654-01 (D.J.R.).

\section{Author Disclosure Statement}

No competing financial interests exist.

\section{References}

1. Bornstein, P. Diversity of function is inherent in matricellular proteins: an appraisal of thrombospondin 1. $J$. Cell Biol. 130:503-506, 1995.

2. Bornstein, P., and Sage, E.H. Matricellular proteins: extracellular modulators of cell function. Curr. Opin. Cell Biol. 14:608-616, 2002.

3. Brekken, R.A., et al. Expression and characterization of murine hevin (SC1), a member of the SPARC family of matricellular proteins. J. Histochem. Cytochem. 52:735-748, 2004. 
4. Chiquet-Ehrismann, R. Tenascins, a growing family of extracellular matrix proteins. Experientia. 51:853-862, 1995.

5. Sage, E.H., and Bornstein, P. Extracellular proteins that modulate cell-matrix interactions. SPARC, tenascin, and thrombospondin. J. Biol. Chem. 266:14831-14834, 1991.

6. Rhee, D.J., Haddadin, R.I., Kang, M.H., and Oh, D.J. Matricellular proteins in the trabecular meshwork. Exp. Eye Res. 88:694-703, 2009.

7. Termine, J.D., et al. Osteonectin, a bone-specific protein linking mineral to collagen. Cell. 26:99-105, 1981.

8. Yan, Q., and Sage, E.H. SPARC, a matricellular glycoprotein with important biological functions. J. Histochem. Cytochem. 47:1495-1506, 1999.

9. Holland, P.W., Harper, S.J., McVey, J.H., and Hogan, B.L. In vivo expression of mRNA for the $\mathrm{Ca}++$-binding protein SPARC (osteonectin) revealed by in situ hybridization. J. Cell Biol. 105:473-482, 1987.

10. Sage, H., Vernon, R.B., Decker, J., Funk, S., and IruelaArispe, M.L. Distribution of the calcium-binding protein SPARC in tissues of embryonic and adult mice. J. Histochem. Cytochem. 37:819-829, 1989.

11. Brekken, R.A., and Sage, E.H. SPARC, a matricellular protein: at the crossroads of cell-matrix. Matrix Biol. 19:569-580, 2000.

12. Bassuk, J.A., et al. Disruption of the Sparc locus in mice alters the differentiation of lenticular epithelial cells and leads to cataract formation. Exp. Eye Res. 68:321-331, 1999.

13. Funk, S.E., and Sage, E.H. The Ca2(+)-binding glycoprotein SPARC modulates cell cycle progression in bovine aortic endothelial cells. Proc. Natl. Acad. Sci. U. S. A. 88:2648-2652, 1991.

14. Funk, S.E., and Sage, E.H. Differential effects of SPARC and cationic SPARC peptides on DNA synthesis by endothelial cells and fibroblasts. J. Cell. Physiol. 154:53-63, 1993.

15. Hasselaar, P., Loskutoff, D.J., Sawdey, M., and Sage, E.H. SPARC induces the expression of type 1 plasminogen activator inhibitor in cultured bovine aortic endothelial cells. J. Biol. Chem. 266:13178-13184, 1991.

16. Lane, T.F., and Sage, E.H. Functional mapping of SPARC: peptides from two distinct $\mathrm{Ca}+(+)$-binding sites modulate cell shape. J. Cell Biol. 111:3065-3076, 1990.

17. Murphy-Ullrich, J.E., Lane, T.F., Pallero, M.A., and Sage, E.H. SPARC mediates focal adhesion disassembly in endothelial cells through a follistatin-like region and the $\mathrm{Ca}(2+)-$ binding EF-hand. J. Cell. Biochem. 57:341-350, 1995.

18. Sage, H., Vernon, R.B., Funk, S.E., Everitt, E.A., and Angello, J. SPARC, a secreted protein associated with cellular proliferation, inhibits cell spreading in vitro and exhibits $\mathrm{Ca}+2$-dependent binding to the extracellular matrix. J. Cell Biol. 109:341-356, 1989.

19. Tremble, P.M., Lane, T.F., Sage, E.H., and Werb, Z. SPARC, a secreted protein associated with morphogenesis and tissue remodeling, induces expression of metalloproteinases in fibroblasts through a novel extracellular matrixdependent pathway. J. Cell Biol. 121:1433-1444, 1993.

20. Maillard, C., Malaval, L., and Delmas, P.D. Immunological screening of SPARC/Osteonectin in nonmineralized tissues. Bone. 13:257-264, 1992.

21. Mann, K., Deutzmann, R., Paulsson, M., and Timpl, R. Solubilization of protein BM-40 from a basement membrane tumor with chelating agents and evidence for its identity with osteonectin and SPARC. FEBS Lett. 218: 167-172, 1987.
22. Allanore, Y., Wipff, J., Kahan, A., and Boileau, C. Genetic basis for systemic sclerosis. Joint Bone Spine. 74:577-583, 2007.

23. Blazejewski, S., et al. Osteonectin (SPARC) expression in human liver and in cultured human liver myofibroblasts. Am. J. Pathol. 151:651-657, 1997.

24. Delany, A.M., McMahon, D.J., Powell, J.S., Greenberg, D.A., and Kurland, E.S. Osteonectin/SPARC polymorphisms in Caucasian men with idiopathic osteoporosis. Osteoporos. Int. 19:969-978, 2008.

25. Framson, P.E., and Sage, E.H. SPARC and tumor growth: where the seed meets the soil? J. Cell. Biochem. 92:679-690, 2004.

26. Frizell, E., et al. Expression of SPARC in normal and fibrotic livers. Hepatology. 21:847-854, 1995.

27. Nakken, K.E., et al. Multiple inflammatory-, tissue remodelling- and fibrosis genes are differentially transcribed in the livers of Abcb4 (-/-) mice harbouring chronic cholangitis. Scand. J. Gastroenterol. 42:1245-1255, 2007.

28. Pichler, R.H., et al. SPARC is expressed in renal interstitial fibrosis and in renal vascular injury. Kidney Int. 50:1978-1989, 1996.

29. Zhou, X., Tan, F.K., Guo, X., and Arnett, F.C. Attenuation of collagen production with small interfering RNA of SPARC in cultured fibroblasts from the skin of patients with scleroderma. Arthritis Rheum. 54:2626-2631, 2006.

30. Zhou, X.D., Xiong, M.M., Tan, F.K., Guo, X.J., and Arnett, F.C. SPARC, an upstream regulator of connective tissue growth factor in response to transforming growth factor beta stimulation. Arthritis Rheum. 54:3885-3889, 2006.

31. Barker, T.H., et al. SPARC regulates extracellular matrix organization through its modulation of integrin-linked kinase activity. J. Biol. Chem. 280:36483-36493, 2005.

32. Barker, T.H., et al. Matricellular homologs in the foreign body response: hevin suppresses inflammation, but hevin and SPARC together diminish angiogenesis. Am. J. Pathol. 166:923-933, 2005.

33. Bradshaw, A.D., et al. SPARC-null mice display abnormalities in the dermis characterized by decreased collagen fibril diameter and reduced tensile strength. J. Invest. Dermatol. 120:949-955, 2003.

34. Bradshaw, A.D., Reed, M.J., and Sage, E.H. SPARC-null mice exhibit accelerated cutaneous wound closure. $J$. Histochem. Cytochem. 50:1-10, 2002.

35. Delany, A.M., et al. Osteopenia and decreased bone formation in osteonectin-deficient mice. J. Clin. Invest. 105: 1325, 2000.

36. Kingsley, D.M. The TGF-beta superfamily: new members, new receptors, and new genetic tests of function in different organisms. Genes Dev. 8:133-146, 1994.

37. Reed, M.J., Vernon, R.B., Abrass, I.B., and Sage, E.H. TGF-beta 1 induces the expression of type I collagen and SPARC, and enhances contraction of collagen gels, by fibroblasts from young and aged donors. J. Cell. Physiol. 158:169-179, 1994.

38. Wrana, J.L., et al. Regulation of transformation-sensitive secreted phosphoprotein (SPPI/osteopontin) expression by transforming growth factor-beta. Comparisons with expression of SPARC (secreted acidic cysteine-rich protein). Biochem. J. 273 Pt 3:523-531, 1991.

39. Kang, M.H., Oh, D.J., Kang, J.H., and Rhee, D.J. Regulation of SPARC by transforming growth factor beta2 in human trabecular meshwork. Invest. Ophthalmol. Vis. Sci. 54:2523-2532, 2013. 
40. Swaminathan, S.S., et al. TGF-beta2-mediated ocular hypertension is attenuated in SPARC-null mice. Invest. Ophthalmol. Vis. Sci. 55:4084-4097, 2014.

41. Francki, A., et al. SPARC regulates the expression of collagen type I and transforming growth factor-beta1 in mesangial cells. J. Biol. Chem. 274:32145-32152, 1999.

42. Francki, A., et al. SPARC regulates TGF-beta1-dependent signaling in primary glomerular mesangial cells. J. Cell. Biochem. 91:915-925, 2004.

43. Picht, G., Welge-Luessen, U., Grehn, F., and LutjenDrecoll, E. Transforming growth factor beta 2 levels in the aqueous humor in different types of glaucoma and the relation to filtering bleb development. Graefes Arch. Clin. Exp. Ophthalmol. 239:199-207, 2001.

44. Tripathi, R.C., Li, J., Chan, W.F., and Tripathi, B.J. Aqueous humor in glaucomatous eyes contains an increased level of TGF-beta 2. Exp. Eye Res. 59:723-727, 1994.

45. Mason, I.J., et al. Developmental and transformationsensitive expression of the Sparc gene on mouse chromosome 11. EMBO J. 5:1831-1837, 1986.

46. Schwarzbauer, J.E., and Spencer, C.S. The Caenorhabditis elegans homologue of the extracellular calcium binding protein SPARC/osteonectin affects nematode body morphology and mobility. Mol. Biol. Cell. 4:941-952, 1993.

47. Sage, H., Johnson, C., and Bornstein, P. Characterization of a novel serum albumin-binding glycoprotein secreted by endothelial cells in culture. J. Biol. Chem. 259:3993-4007, 1984.

48. Maurer, P., et al. High-affinity and low-affinity calcium binding and stability of the multidomain extracellular 40$\mathrm{kDa}$ basement membrane glycoprotein (BM-40/SPARC/ osteonectin). Eur. J. Biochem. 205:233-240, 1992.

49. Romberg, R.W., Werness, P.G., Lollar, P., Riggs, B.L., and Mann, K.G. Isolation and characterization of native adult osteonectin. J. Biol. Chem. 260:2728-2736, 1985.

50. Kaufmann, B., et al. Structural variability of BM-40/ SPARC/osteonectin glycosylation: implications for collagen affinity. Glycobiology. 14:609-619, 2004.

51. Kelm Jr., R.J., and Mann, K.G. The collagen binding specificity of bone and platelet osteonectin is related to differences in glycosylation. J. Biol. Chem. 266:96329639, 1991.

52. Sasaki, T., Hohenester, E., Gohring, W., and Timpl, R. Crystal structure and mapping by site-directed mutagenesis of the collagen-binding epitope of an activated form of BM-40/SPARC/osteonectin. EMBO J. 17:1625-1634, 1998.

53. Sasaki, T., et al. Limited cleavage of extracellular matrix protein BM-40 by matrix metalloproteinases increases its affinity for collagens. J. Biol. Chem. 272:9237-9243, 1997.

54. Engel, J., Taylor, W., Paulsson, M., Sage, H., and Hogan, B. Calcium binding domains and calcium-induced conformational transition of SPARC/BM-40/osteonectin, an extracellular glycoprotein expressed in mineralized and nonmineralized tissues. Biochemistry. 26:6958-6965, 1987.

55. Pottgiesser, J., et al. Changes in calcium and collagen IV binding caused by mutations in the EF hand and other domains of extracellular matrix protein BM-40 (SPARC, osteonectin). J. Mol. Biol. 238:563-574, 1994.

56. Chlenski, A., et al. Secreted protein acidic and rich in cysteine is a matrix scavenger chaperone. PLoS One. 6:e23880, 2011.

57. Emerson, R.O., Sage, E.H., Ghosh, J.G., and Clark, J.I. Chaperone-like activity revealed in the matricellular protein SPARC. J. Cell. Biochem. 98:701-705, 2006.
58. Yan, Q., Sage, E.H., and Hendrickson, A.E. SPARC is expressed by ganglion cells and astrocytes in bovine retina. J. Histochem. Cytochem. 46:3-10, 1998.

59. Friedman, D.S., et al. Prevalence of open-angle glaucoma among adults in the United States. Arch. Ophthalmol. 122:532-538, 2004.

60. Quigley, H.A. Number of people with glaucoma worldwide. Br. J. Ophthalmol. 80:389-393, 1996.

61. Quigley, H.A., and Broman, A.T. The number of people with glaucoma worldwide in 2010 and 2020. Br. J. Ophthal. 90:262-267, 2006.

62. Nemesure, B., et al. Incident open-angle glaucoma and intraocular pressure. Ophthalmology. 114:1810-1815, 2007.

63. Barany, E.H., and Scotchbrook, S. Influence of testicular hyaluronidase on the resistance to flow through the angle of the anterior chamber. Acta Physiol. Scand. 30:240-248, 1954.

64. Bradley, J.M., et al. Effect of matrix metalloproteinases activity on outflow in perfused human organ culture. Invest. Ophthal. Vis. Sci. 39:2649-2658, 1998.

65. Keller, K.E., Bradley, J.M., Kelley, M.J., and Acott, T.S. Effects of modifiers of glycosaminoglycan biosynthesis on outflow facility in perfusion culture. Invest. Ophthalmol. Vis. Sci. 49:2495-2505, 2008.

66. Knepper, P.A., Farbman, A.I., and Telser, A.G. Exogenous hyaluronidases and degradation of hyaluronic acid in the rabbit eye. Invest. Ophthalmol. Vis. Sci. 25:286-293, 1984.

67. Larsson, L.I., Rettig, E.S., and Brubaker, R.F. Aqueous flow in open-angle glaucoma. Arch. Ophthalmol. 113: 283-286, 1995.

68. Sage, E.H., Terms of attachment: SPARC and tumorigenesis. Nat. Med. 3:144-146, 1997.

69. Weinreb, R.N., Toris, C.B., Gabelt, B.T., Lindsey, J.D., and Kaufman, P.L. Effects of prostaglandins on the aqueous humor outflow pathways. Surv. Ophthalmol. 47 Suppl 1:S53-S64, 2002.

70. Weinreb, R.N. Uveoscleral outflow: the other outflow pathway. J. Glaucoma. 9:343-345, 2000.

71. Schachtschabel, U., Lindsey, J.D., and Weinreb, R.N. The mechanism of action of prostaglandins on uveoscleral outflow. Curr. Opin. Ophthalmol. 11:112-115, 2000.

72. Maepea, O., and Bill, A. Pressures in the juxtacanalicular tissue and Schlemm's canal in monkeys. Exp. Eye Res. 54:879-883, 1992.

73. Chatterjee, A., Villarreal Jr., G., and Rhee, D.J. Matricellular proteins in the trabecular meshwork: review and update. J. Ocul. Pharmacol. Ther. 30:447-463, 2014.

74. Gottanka, J., Johnson, D.H., Martus, P., and Lutjen-Drecoll, E. Severity of optic nerve damage in eyes with POAG is correlated with changes in the trabecular meshwork. J. Glaucoma. 6:123-132, 1997.

75. Ueda, J., Wentz-Hunter, K., and Yue, B.Y. Distribution of myocilin and extracellular matrix components in the juxtacanalicular tissue of human eyes. Invest. Ophthalmol. Vis. Sci. 43:1068-1076, 2002.

76. Last, J.A., et al. Elastic modulus determination of normal and glaucomatous human trabecular meshwork. Invest. Ophthalmol. Vis. Sci. 52:2147-2152, 2011.

77. Vittal, V., Rose, A., Gregory, K.E., Kelley, M.J., and Acott, T.S. Changes in gene expression by trabecular meshwork cells in response to mechanical stretching. Invest. Ophthalmol. Vis. Sci. 46:2857-2868, 2005.

78. Tomarev, S.I., Wistow, G., Raymond, V., Dubois, S., and Malyukova, I. Gene expression profile of the human trabecular meshwork: NEIBank sequence tag analysis. Invest. Ophthalmol. Vis. Sci. 44:2588-2596, 2003. 
79. Rhee, D.J., Fariss, R.N., Brekken, R., Sage, E.H., and Russell, P. The matricellular protein SPARC is expressed in human trabecular meshwork. Exp. Eye Res. 77:601-607, 2003.

80. Tomarev, S.I. Eyeing a new route along an old pathway. Nat. Med. 7:294-295, 2001.

81. Thomasy, S.M., Wood, J.A., Kass, P.H., Murphy, C.J., and Russell, P. Substratum stiffness and latrunculin B regulate matrix gene and protein expression in human trabecular meshwork cells. Invest. Ophthalmol. Vis. Sci. 53:952-958, 2012.

82. Haddadin, R.I., et al. SPARC-null mice exhibit lower intraocular pressures. Invest. Ophthalmol. Vis. Sci. 50: 3771-3777, 2009.

83. Swaminathan, S.S., et al. Secreted protein acidic and rich in cysteine (SPARC)-null mice exhibit more uniform outflow. Invest. Ophthalmol. Vis. Sci. 54:2035-2047, 2013.

84. Swaminathan, S.S., Oh, D.J., Kang, M.H., and Rhee, D.J. Aqueous outflow: segmental and distal flow. J. Cataract Refract. Surg. 40:1263-1272, 2014.

85. Oh, D.J., et al. Overexpression of SPARC in human trabecular meshwork increases intraocular pressure and alters extracellular matrix. Invest. Ophthalmol. Vis. Sci. 54:3309-3319, 2013.

86. Kudo, H., Hirayoshi, K., Kitagawa, Y., Imamura, S., and Nagata, K. Two collagen-binding proteins, osteonectin and HSP47, are coordinately induced in transformed keratinocytes by heat and other stresses. Exp. Cell Res. 212:219-224, 1994.

87. Neri, M., Descalzi-Cancedda, F., and Cancedda, R. Heatshock response in cultured chick embryo chondrocytes. Osteonectin is a secreted heat-shock protein. Eur. J. Biochem. 205:569-574, 1992.

88. Chatterjee, A., Kang, M., Oh, D., and Rhee, D.J. Incubation with SPARC mitigates MMP-2-mediated collagen 4 degradation in vitro. Invest. Ophthalmol. Vis. Sci. 55:5685, 2014.

89. Bhoopathi, P., et al. The role of MMP-9 in the anti-angiogenic effect of secreted protein acidic and rich in cysteine. Br. J. Cancer. 102:530-540, 2010.

90. Ochiai, Y., and Ochiai, H. Higher concentration of transforming growth factor-beta in aqueous humor of glaucomatous eyes and diabetic eyes. Jpn. J. Ophthalmol. 46:249-253, 2002.

91. Bataller, R., and Brenner, D.A. Liver fibrosis. J. Clin. Invest. 115:209-218, 2005.

92. Huggins, J.T., and Sahn, S.A. Causes and management of pleural fibrosis. Respirology. 9:441-447, 2004.

93. Liu, Y. Renal fibrosis: new insights into the pathogenesis and therapeutics. Kidney Int. 69:213-217, 2006.

94. Fuchshofer, R., Yu, A.H., Welge-Lussen, U., and Tamm, E.R. Bone morphogenetic protein-7 is an antagonist of transforming growth factor-beta2 in human trabecular meshwork cells. Invest. Ophthalmol. Vis. Sci. 48:715-726, 2007.

95. Li, G., et al. Sustained stress response after oxidative stress in trabecular meshwork cells. Mol. Vis. 13:2282-2288, 2007.

96. Zhao, X., Ramsey, K.E., Stephan, D.A., and Russell, P. Gene and protein expression changes in human trabecular meshwork cells treated with transforming growth factorbeta. Invest. Ophthalmol. Vis. Sci. 45:4023-4034, 2004.

97. Zhao, X., and Russell, P. Versican splice variants in human trabecular meshwork and ciliary muscle. Mol. Vis. 11:603-608, 2005.

98. Fleenor, D.L., et al. TGFbeta2-induced changes in human trabecular meshwork: implications for intraocular pressure. Invest. Ophthalmol. Vis. Sci. 47:226-234, 2006.
99. Gottanka, J., Chan, D., Eichhorn, M., Lutjen-Drecoll, E., and Ethier, C.R. Effects of TGF-beta2 in perfused human eyes. Invest. Ophthalmol. Vis. Sci. 45:153-158, 2004.

100. Bollinger, K.E., et al. Quantitative proteomics: TGFbe$\mathrm{ta}(2)$ signaling in trabecular meshwork cells. Invest. Ophthalmol. Vis. Sci. 52:8287-8294, 2011.

101. Norose, K., et al. SPARC deficiency leads to early-onset cataractogenesis. Invest. Ophthalmol. Vis. Sci. 39:2674-2680, 1998.

102. Norose, K., Lo, W.K., Clark, J.I., Sage, E.H., and Howe, C.C. Lenses of SPARC-null mice exhibit an abnormal cell surface-basement membrane interface. Exp. Eye Res. 71:295-307, 2000.

103. Weaver, M.S., Toida, N., and Sage, E.H. Expression of integrin-linked kinase in the murine lens is consistent with its role in epithelial-mesenchymal transition of lens epithelial cells in vitro. Mol. Vis. 13:707-718, 2007.

104. Uga, S., Kohara, M., and Ishikawa, S. Morphological study of age-related changes in mouse lens. Jpn. J. Ophthalmol. 27:157-165, 1983.

105. Seland, J.H. Ultrastructural changes in the normal human lens capsule from birth to old age. Acta Ophthalmol. 52:688-706, 1974.

106. Sawhney, R.S. Identification of SPARC in the anterior lens capsule and its expression by lens epithelial cells. Exp. Eye Res. 61:645-648, 1995.

107. Kuszak, J.R., et al. The internalization of posterior subcapsular cataracts (PSCs) in Royal College of Surgeons (RCS) rats. II. The inter-relationship of optical quality and structure as a function of age. Mol. Vis. 5:7, 1999.

108. Blakely, E.A., et al. Growth and differentiation of human lens epithelial cells in vitro on matrix. Invest. Ophthalmol. Vis. Sci. 41:3898-3907, 2000.

109. Menko, S., Philp, N., Veneziale, B., and Walker, J. Integrins and development: how might these receptors regulate differentiation of the lens. Ann. N. Y. Acad. Sci. 842: 36-41, 1998.

110. Yan, Q., Clark, J.I., and Sage, E.H. Expression and characterization of SPARC in human lens and in the aqueous and vitreous humors. Exp. Eye Res. 71:81-90, 2000.

111. Yan, Q., et al. Alterations in the lens capsule contribute to cataractogenesis in SPARC-null mice. J. Cell Sci. 115: 2747-2756, 2002.

112. Schulz, M.W., Chamberlain, C.G., de Iongh, R.U., and McAvoy, J.W. Acidic and basic FGF in ocular media and lens: implications for lens polarity and growth patterns. Development. 118:117-126, 1993.

113. Hyatt, G.A., and Beebe, D.C. Regulation of lens cell growth and polarity by an embryo-specific growth factor and by inhibitors of lens cell proliferation and differentiation. Development. 117:701-709, 1993.

114. Yan, Q., Clark, J.I., Wight, T.N., and Sage, E.H. Alterations in the lens capsule contribute to cataractogenesis in SPARC-null mice. J. Cell Sci. 115:2747-2756, 2002.

115. Kantorow, M., et al. Increased expression of osteonectin/ SPARC mRNA and protein in age-related human cataracts and spatial expression in the normal human lens. Mol. Vis. 6:24-29, 2000.

116. Kantorow, M., Horwitz, J., and Carper, D. Up-regulation of osteonectin/SPARC in age-related cataractous human lens epithelia. Mol. Vis. 4:17, 1998.

117. Mendis, D.B., Malaval, L., and Brown, I.R. SPARC, an extracellular matrix glycoprotein containing the follistatin module, is expressed by astrocytes in synaptic enriched regions of the adult brain. Brain Res. 676:69-79, 1995. 
118. Mendis, D.B., and Brown, I.R. Expression of the gene encoding the extracellular matrix glycoprotein SPARC in the developing and adult mouse brain. Brain Res. Mol. Brain Res. 24:11-19, 1994.

119. Kim, S.Y., et al. Spatiotemporal distribution of SPARC/ osteonectin in developing and mature chicken retina. Exp. Eye Res. 65:681-689, 1997.

120. Guermah, M., et al. Transcription of a quail gene expressed in embryonic retinal cells is shut off sharply at hatching. Proc. Natl. Acad. Sci. U. S. A. 88:4503-4507, 1991.

121. Rodriguez, I.R., Moreira, E.F., Bok, D., and Kantorow, M. Osteonectin/SPARC secreted by RPE and localized to the outer plexiform layer of the monkey retina. Invest. Ophthalmol. Vis. Sci. 41:2438-2444, 2000.

122. Anderson, D.H., Guerin, C.J., Hageman, G.S., Pfeffer, B.A., and Flanders, K.C. Distribution of transforming growth factor-beta isoforms in the mammalian retina. $J$. Neurosci. Res. 42:63-79, 1995.

123. Kucukdereli, H., et al. Control of excitatory CNS synaptogenesis by astrocyte-secreted proteins Hevin and SPARC. Proc. Natl. Acad. Sci. U. S. A. 108:E440-E449, 2011.

124. Ratnayaka, A., et al. Trafficking of osteonectin by retinal pigment epithelial cells: evidence for basolateral secretion. Int. J. Biochem. Cell Biol. 39:85-92, 2007.

125. Magee, R.M., et al. Synthesis of osteonectin by human retinal pigment epithelial cells is modulated by cell density. Invest. Ophthalmol. Vis. Sci. 41:2707-2711, 2000.

126. Hiscott, P., Sheridan, C., Magee, R.M., and Grierson, I. Matrix and the retinal pigment epithelium in proliferative retinal disease. Prog.Retin. Eye Res. 18:167-190, 1999.

127. Grierson, I., et al. Development, repair and regeneration of the retinal pigment epithelium. Eye. 8 Pt 2:255-262, 1994.

128. Hiscott, P., et al. Pathobiology of epiretinal and subretinal membranes: possible roles for the matricellular proteins thrombospondin 1 and osteonectin (SPARC). Eye. 16: 393-403, 2002.

129. Robey, H.L., Hiscott, P.S., and Grierson, I. Cytokeratins and retinal epithelial cell behaviour. J. Cell Sci. $102 \mathrm{Pt}$ 2:329-340, 1992.
130. Hagan, S., et al. Effects of the matricellular protein SPARC on human retinal pigment epithelial cell behavior. Mol. Vis. 9:87-92, 2003.

131. Hiscott, P., Gray, R., Grierson, I., and Gregor, Z. Cytokeratin-containing cells in proliferative diabetic retinopathy membranes. Br. J. Ophthalmol. 78:219-222, 1994.

132. An, E., et al. Secreted proteome profiling in human RPE cell cultures derived from donors with age related macular degeneration and age matched healthy donors. J. Proteome Res. 5:2599-2610, 2006.

133. Bampton, E.T., Ma, C.H., Tolkovsky, A.M., and Taylor, J.S. Osteonectin is a Schwann cell-secreted factor that promotes retinal ganglion cell survival and process outgrowth. Eur. J. Neurosci. 21:2611-2623, 2005.

134. Ma, C.H., Bampton, E.T., Evans, M.J., and Taylor, J.S. Synergistic effects of osteonectin and brain-derived neurotrophic factor on axotomized retinal ganglion cells neurite outgrowth via the mitogen-activated protein kinase-extracellular signal-regulated kinase 1/2 pathways. Neuroscience. 165:463-474, 2010.

135. Piri, N., Kwong, J.M., Song, M., Elashoff, D., and Caprioli, J. Gene expression changes in the retina following optic nerve transection. Mol. Vis. 12:1660-1673, 2006.

Received: May 3, 2015

Accepted: June 15, 2015

Address correspondence to:

Dr. Douglas J. Rhee

Department of Ophthalmology and Visual Sciences University Hospitals Eye Institute Case Western Reserve University School of Medicine 11100 Euclid Avenue WRN 5068

Cleveland, $\mathrm{OH} 44106$

E-mail: dougrhee@aol.com 